\title{
Response of Potato Productivity and Storability to some Potassium Levels and Foliar Spray with some Macro and Microelements Hala A. El-Sayed ${ }^{1}$; A. H. A. El-Morsy ${ }^{2}$; H. A. El-Bauome' ${ }^{1}$ and H. R. Moharam ${ }^{1}$ ${ }^{1}$ Veg. And Flori. Dept., Fac. Of Agric., Mans. Univ., Egyet. \\ ${ }^{2}$ Veg. Res. Dept., Hort. Res. Inst., Agric. Res.Center, Giza, Egypt.
}

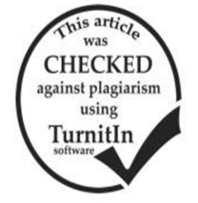

\section{ABSTRACT}

Two field trials were conducted on potato plants cv. Spunta, in vegetable private farm at Al-Enaniya village, Sinbelawin district, Dakahlia Governoraten, during 2014/2015 and 2015/2016 seasons to study the effect of soil application of potassium levels (96, 72 and $48 \mathrm{~kg} \mathrm{~K}_{2} \mathrm{O} / \mathrm{fed}$ ), either single and/or in combination with foliar spray of macro and micronutrients compound ( $\mathrm{N}=25 \%, \mathrm{P}=16 \%, \mathrm{~K}=12 \%$, $\mathrm{Zn}=0.5 \%, \mathrm{Fe}=0.5 \%, \mathrm{Mn}=0.5 \%, \mathrm{Cu}=0.3 \%$ ) at different levels i.e., 6,4 and $2 \mathrm{~g} / \mathrm{L}$ on plant growth, yield and its components, as well as chemical constituents and storability of tubers. In general, results showed that potato plants fertilized with potassium sulfate at the level of $96 \mathrm{~kg} \mathrm{~K}_{2} \mathrm{O} /$ fed significantly increased plant stem length, number of leaves, leaf area and foliage plant fresh and dry weights as well as total yield, number of tubers/plant and tuber weight/plant, Moreover, this level of potassium significantly increased TSS, dry matter, specific gravity, Ascorbic acid (mg/g FW) and concentrations of macro and micronutrients (N, P, K, Fe, Zn, Mn, Cu). Also, potassium fertilization at of $96 \mathrm{~kg} \mathrm{~K}_{2} \mathrm{O} / \mathrm{fed}$ had the most interesting observation which enhancing storability during storage period. Foliar spray with macro and micro nutrients at a rate of $6 \mathrm{~g} / \mathrm{L}$ resulted in an increases in the most vegetative growth parameters, yield and tubers quality traits. However, weight loss percentage of tubers was significantly reduced during storage period.The combined treatments of potassium levels and foliar spray with some macro and micronutrients were generally more effective on the most studied parameters than single ones. The best results were obtained by application $96 \mathrm{~kg} \mathrm{~K} 2 \mathrm{O} / \mathrm{fed}$ with foliar spray of macro and micronutrients at the rate of $6 \mathrm{~g} / \mathrm{L}$. This treatment achieved increases in vegetative growth characters, total tubers yield (ton/fed), ascorbic acid (mg/g FW), TSS\% of tuber, concentrations of $\mathrm{N}, \mathrm{P}, \mathrm{K}$, micronutrients $(\mathrm{Fe}, \mathrm{Zn}, \mathrm{Mn}$ and $\mathrm{Cu})$ in tubers and enhanced the tubers storability comparing with the other ones. Therefore, this treatment could be recommended for raising potato yield and improving tuber quality during storage period under similar conditions to this work.

Keywords: Potato, Potassium levels, Solanum tuberosum L., Tuber production and Top dressing.

\section{INTRODUCTION}

Potato (Solanum tuberosum, L.) is a major world food crop. Potato is exceeded only by wheat, rice, and maize in world production for human consumption. In Egypt, it has been generally cultivated for both local consumption and export. Therefore, increasing potato yield and improving tuber quality are essential aims for both growers and consumers, but it usually depends on many factors especially that influence the plant growth throughout the growth period.

Potassium is involved in many processes within the plant as a catalyst. It plays a role in carbohydrate synthesis and translocation, enhances $\mathrm{N}$ uptake, and promotes protein synthesis (Marschner, 1995). Morever, Potassium is an essential plant nutrient that plays a very important role in plant growth and development. Its role is well documented in photosynthesis, increasing enzyme activity, improving synthesis of protein, carbohydrates and fats, translocation of photosynthetic, enabling their ability to resist pests and diseases (Regmi et al, 2002).

Potato being a high nutrient mining crop it needs a higher amount of $\mathrm{K}$ for its economic tuber production (Regmi et al, 2002). In this regard, El- Sawy et al. (2000), Singh and Bansal (2000), Allison et al. (2001) and El-Hadidi and Mansour (2008) showed that the most vegetative parameters, yield and its components were significantly increased with increasing the applied K-levels from 48 to 96 $\mathrm{kg} \mathrm{K}_{2} \mathrm{O}$ /fed. Moreover, several investigators reported that potato plants growth, yield and storability were improved by potassium application (El-Sirafy et al., 2008; Mahmoud and Hafez, 2010; Kumar et al., 2010 and Abd El-Latif et al., 2011). Regarding to foliar spray with macro and micronutrients, the efficiency of fertilizers used in Egypt is low, either as a result of high $\mathrm{pH}$ of soil or high concentration of soil calcium carbonate. This problem could be solved by soil addition of great amounts of macro and micro-elements fertilizers or through foliar application
(Abdel-Hadi et al, 1986). The positive effect of foliar application of macro and micronutrients on growth, yield and chemical constituents of different plants may be attributed to these elements which can be readily absorbed by the leaves as a result of foliar spraying application and not lost through fixation, decomposition or leaching under unfavorable soils conditions (Doeing, 1986). Several attempts were done on the application of micronutrients spray to correct deficiency symptoms and enhance the vegetative growth of potato which in turn reflects on increasing yield and its quality for facing local consumption and exportation (Stashauskaite et al., 1979; Medvedv et al., 1981; Rashid et al., 1989 and Taya et al., 1994). Several investigators indicated that spraying plants with macronutrients enhanced plant growth, stimulated dry matter accumulation and increased yield and quality as well as chemical composition (Marchand et al., 1999; Roemheld and El-Fouly, 1999; Ewais et al., 2010; Habib et al., 2011; Eleiwa et al., 2012; Salim et al., 2014 and Ghosh et al., 2017).

Therefore, the aim of the study was to investigate the effect of soil application of potassium levels and foliar spray with some macro and micronutrients levels in addition to their interactions on potato productivity and storability under the conditions of North Delta region, Dakahlia Governorate.

\section{MATERIALS AND METHODS}

A field experiment on potato plants cv. Spunta, in the vegetable private farm at Al-Enaniya village, Sinbelawin district, Dakahlia Governoraten, loam soil during two winter seasons (2014/2015 and 2015/2016) to study the effect of some potassium levels, either single and/or in combination with foliar spray of macro and micronutrients obtained from commercial compound called Estema green on plant growth, yield and its components, as well as chemical constituents and storability of tubers. 
The experiment layout was split plot system in a randomized complete block design with three replicates. Potassium levels occupied the main plots which were subdivided to 4 sub plots each contained one of the foliar spray of macro and micronutrients (Stema green) rates. The plot area was $17.5 \mathrm{~m}^{2}$ (1/240 feddan) which contained 5 ridges, each $5 \mathrm{~m}$ long and $0.7 \mathrm{~m}$ width. Each experiment included 12 treatments which were 3 levels of potassium and 4 rates of foliar spray macro micronutrients as follows:

a- soil potassium levels:

1- $96 \mathrm{~kg} \mathrm{~K}_{2} \mathrm{O}_{5} /$ fed.

2- $72 \mathrm{~kg} \mathrm{~K}_{2} \mathrm{O}_{5} /$ fed.

3- $48 \mathrm{~kg} \mathrm{~K}_{2} \mathrm{O}_{5} /$ fed.

b- Foliar spray rates of macro and micronutrients:

1- Control treatment (water).

2- Foliar spray with $2 \mathrm{~g} / \mathrm{L}$.

3- Foliar spray with $4 \mathrm{~g} / \mathrm{L}$.

4- Foliar spray with $6 \mathrm{~g} / \mathrm{L}$.

In both growing seasons, foliar spray of macro and micronutrients as a Estema green compound $(\mathrm{N}=25 \%, \quad \mathrm{P}=16 \%, \quad \mathrm{~K}=12 \%, \quad \mathrm{Zn}=0.5 \%, \quad \mathrm{Fe}=0.5 \%$, $\mathrm{Mn}=0.5 \%, \mathrm{Cu}=0.3 \%$ ) and foliar spray at 45,60 and 75 days after planting (DAP).

Tuber seeds were planted on $20^{\text {th }}$ and $15^{\text {th }}$ of October in the first and the second seasons, respectively. All treatments were fertilized with the recommendation rates of Nitrogen at $180 \mathrm{~kg} \mathrm{~N} / \mathrm{fed}$ (ammonium nitrate, $33.5 \% \mathrm{~N}$ ) was added at three equal doses after 3, 5 and 7 weeks from planting and Phosphorus at $75 \mathrm{~kg} \mathrm{P}_{2} \mathrm{O}_{5} /$ fed (Superphosphate $15.5 \% \quad \mathrm{P}_{2} \mathrm{O}_{5}$ ) was added once before planting. Potassium was added at different levels $\left(96,72,48 \mathrm{~kg} \mathrm{~K}_{2} \mathrm{O} / \mathrm{fed}\right)$ as potassium sulphate $\left(48 \% \mathrm{~K}_{2} \mathrm{O}\right)$ after 7 weeks from planting date. The other cultural practices were applied according to the instructions laid down by the Ministry of Agriculture, Egypt.

\section{Data recorded:}

\section{1- Growth parameters:}

A random sample of three potato plants were taken from each plot after 70 and 90 DAP to estimate the plant stem length $(\mathrm{cm})$, number of main stems/plant, number of leaves/plant, leaf area, foliage fresh and dry weights/plant (gm), number of tubers/plant and tubers weight/plant (gm).

\section{2- Yield and its components:}

At harvest time, yield of each plot was weighted in $\mathrm{kg}$ and converted to total yield (ton/fed).

\section{Grading of tubers:}

Tuber from each plot were size - graded to three classes according to tuber diameter, less than $30 \mathrm{~mm}$, from 30 to $60 \mathrm{~mm}$, and over than $60 \mathrm{~mm}$, then each grade was weighed separately and calculated as a percentage of the total yield.

\section{3- Chemical analysis:}

All chemical constituents were determined after harvest in the digested dry matter of tubers. Nitrogen were determined according to Prummel (1978), phosphorus and potassium were determined according to Jackson (1967), iron, zinc, manganese, cupper and ascorbic acid were determined according A.O.A.C (1990). Percentage of total soluble solids (TSS\%) was determined by a hand refractometer.

\section{4- Storability:}

After curing, random samples $(5 \mathrm{~kg}$ of marketable yield from each plot) were taken, stored in case of paper under normal room conditions. The percentage of weight loss was recorded at 60 days' intervals during the storage period (six months).

It was estimated using the following formula: -

Weight loss $(\%)=$ (weight before storage - weight after storage) $X 100$ Tuber weight before storage

Ascorbic acid (mg/g FW) was determined at the end of storage period

Data were subjected to the statistical analysis and means were compared using new L.S.D according to Gomez and Gomez (1984).

\section{RESULTS AND DISCUSSION}

Results are presented under separate heading, include vegetative growth, tuber yield and its components, chemical composition of tuber, quality of tubers and storability. It is hoped that such sequence simplifies our presentation subject, since each part represents an identical stage of plant growth

\section{1- Vegetative growth characters:}

Results in Tables 1 and 2 showed that the highest values of stem length, number of main stems/plant, number of leaves/plant, leaf area, foliage dry and fresh weights/plant, number of tubers/plant and tuber weight of potato were obtained from plants fertilized with the high level of potassium sulphate $\left(96 \mathrm{Kg} \mathrm{K} \mathrm{K}_{2} \mathrm{O} / \mathrm{fed}\right.$ ) followed by $72 \mathrm{Kg}$ $\mathrm{K}_{2} \mathrm{O}$ /fed and $48 \mathrm{Kg} \mathrm{K}_{2} \mathrm{O} /$ fed in both seasons. Similar results were obtained by Mahmoud and Hafez (2010) who found that potato vegetative growth parameters i.e., plant length, fresh and dry weight of leaves and shoots were gradually significantly increased by increasing the level of potassium application from 40, 80 up to $120 \mathrm{~kg} \mathrm{~K}_{2} \mathrm{O} /$ fed.

Also, Kumar et al. (2010) found that $100 \%$ of the recommended potassium fertilizer dose' recorded the best results in respect of plant height, leaf number per plant, number of days for bulb initiation (68.11 days) and number of days for bulb maturity (125.06 days) of onion plant.

Also, the results in Tables 1 and 2 indicate that high significant values of stem length, number of main stems/plant, number of leaves/plant, leaf area, foliage dry and fresh weights/plant, number of tubers/plant and tuber weight of potato were obtained from plants sprayed with macro and micronutrients at the different levels as compared with control (sprayed with water) gradual increases were recorded with increasing spray levels from $2 \mathrm{~g} / \mathrm{l}$ up to $6 \mathrm{~g} / \mathrm{l}$ in both seasons. Similar results were obtained by Dkhil et al. (2011) who showed that foliar fertilizer of potato had a significant effect on plant height, plant fresh and dry weight. Also Salim et al. (2014) found that the potato plants which received foliar application of $2000 \mathrm{ppm}$ potassium nitrate and potassium silicate gave the highest values for plant length as compared with other treatments. Moreover, Ghosh et al. (2017) and Moinuddin et al. (2005) found that when the crop was fertilized by foliar application with micronutrient in combination with NPK enhanced most of the vegetative growth of potato (plant height, number of leaves per plant and number of branches per plant). 
In the same tables, the interaction results indicated that the highest values of vegetative growth characters i.e., stem length, number of main stems/plant, number of leaves/plant, leaf area, foliage dry and fresh weights/plant, number of tubers/plant and tuber weight were significantly obtained by using potassium at $96 \mathrm{~kg} \mathrm{~K}_{2} \mathrm{O} /$ fed and spray with macro and micronutrients at $6 \mathrm{~g} / \mathrm{L}$ compared with the other interactions. Similar results were obtained by Ewais et al. (2010) when used K-fertilizer as $50 \%$ of the recommended rate used as soil application $+50 \%$ of that added as foliar application. Moreover, Eleiwa et al. (2012) found that the interaction between potassium levels and foliar nutritional compounds significantly affected number of leaves/plant.

Table 1. Vegetative growth characters of potato plants as affected by potassium levels, foliar spray with macro and micronutrients and their interactions during 2014/2015 (S1) and 2005/2006 (S2) winter seasons.

\begin{tabular}{|c|c|c|c|c|c|c|c|c|c|c|c|c|c|c|c|c|c|c|c|}
\hline \multirow{3}{*}{$\begin{array}{l}\text { Characters } \\
\text { Treatments }\end{array}$} & \multicolumn{3}{|c|}{$\begin{array}{l}\text { Stem length } \\
\text { (cm) }\end{array}$} & \multicolumn{4}{|c|}{$\begin{array}{l}\text { No. of main } \\
\text { stems/plant }\end{array}$} & \multicolumn{4}{|c|}{$\begin{array}{l}\text { Number of } \\
\text { leaves/plant }\end{array}$} & \multicolumn{4}{|c|}{$\begin{array}{l}\text { Leaf area/plant } \\
\qquad\left(\mathrm{m}^{2}\right)\end{array}$} & \multicolumn{4}{|c|}{$\begin{array}{c}\text { Foliage dry weight } \\
\text { (gm) }\end{array}$} \\
\hline & 70 day & & & & day & & & & day & & & & lay & 90 & & & day & & day \\
\hline & $\begin{array}{ll}\text { S1 } & \text { S2 }\end{array}$ & S1 & S2 & S1 & S2 & S1 & S2 & S1 & S2 & S1 & S2 & S1 & S2 & S1 & S2 & S1 & S2 & S1 & S2 \\
\hline
\end{tabular}

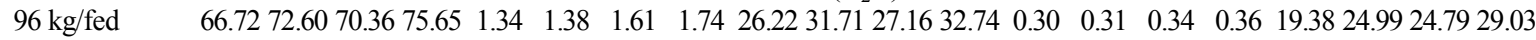

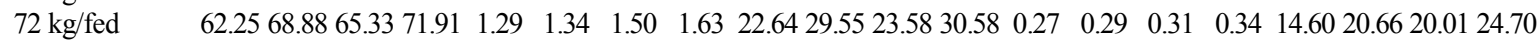

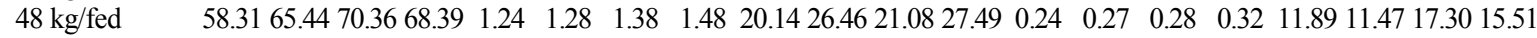

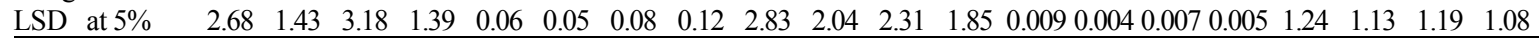
Foliar spray macro and micronutrients.

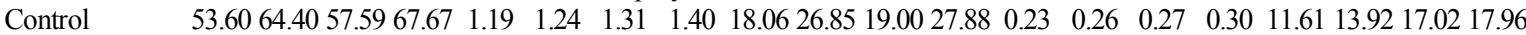

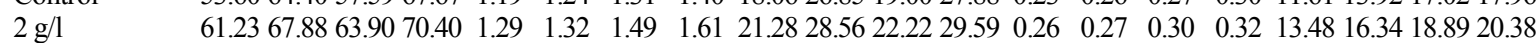

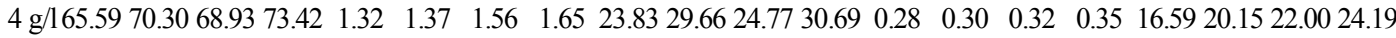

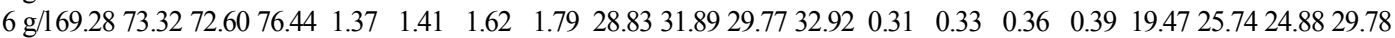
\begin{tabular}{llllllllllllllllllllll} 
LSD at $5 \%$ & 3.66 & 1.61 & 2.80 & 1.57 & 0.05 & 0.05 & 0.11 & 0.09 & 2.11 & 1.23 & 1.75 & 1.09 & 0.14 & 0.06 & 0.01 & 0.09 & 0.78 & 1.34 & 0.67 & 1.29 \\
\hline
\end{tabular} Interactions:K levels\& Foliar spray

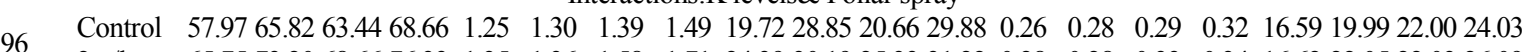

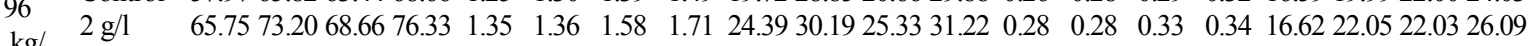

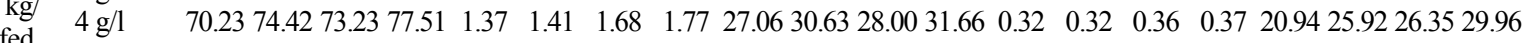

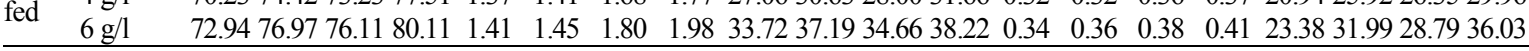

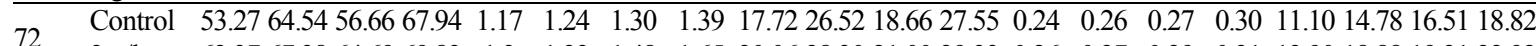

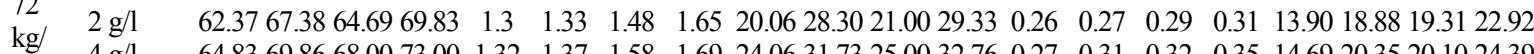

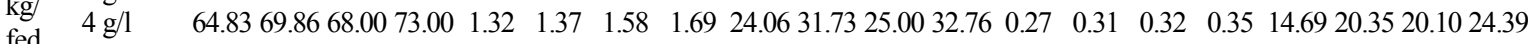

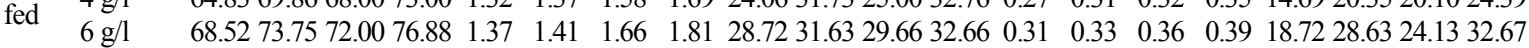

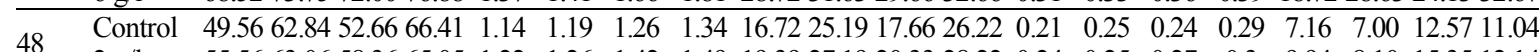

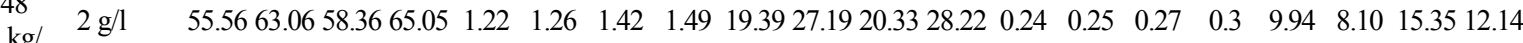

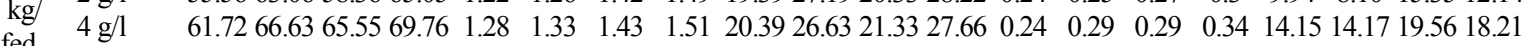

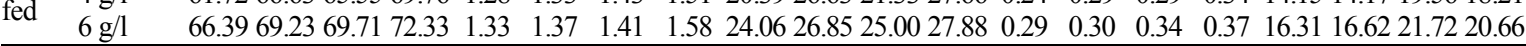
\begin{tabular}{lllllllllllllllllllll}
\hline L.S.D. at 5\% & 6.34 & 4.85 & 4.86 & 2.72 & 0.09 & 0.09 & 0.19 & 0.16 & 3.46 & 2.13 & 3.62 & 2.06 & 0.02 & 0.08 & 0.04 & 0.12 & 1.45 & 2.32 & 1.36 & 1.91 \\
\hline
\end{tabular}

Table 2. Vegetative growth characters of potato plants as affected by potassium levels, foliar spray with macro and micronutrients and their interactions during 2014/2015 (S1) and 2005/2006 (S2) winter seasons.

\begin{tabular}{|c|c|c|c|c|c|c|c|c|c|c|c|c|c|}
\hline \multirow{3}{*}{\multicolumn{2}{|c|}{$\begin{array}{l}\text { Characters } \\
\text { Treatments }\end{array}$}} & \multicolumn{4}{|c|}{ Foliage Fresh Wight (gm /Plant) } & \multicolumn{4}{|c|}{ Number of tubers / plant } & \multicolumn{4}{|c|}{ Tubers weight / plant (gm) } \\
\hline & & \multicolumn{2}{|c|}{70 day } & \multicolumn{2}{|c|}{90 day } & \multicolumn{2}{|c|}{70 day } & \multicolumn{2}{|c|}{90 day } & \multicolumn{2}{|c|}{70 day } & \multicolumn{2}{|c|}{90 day } \\
\hline & & S1 & S2 & S1 & S2 & S1 & $\mathbf{S 2}$ & S1 & $\mathbf{S 2}$ & S1 & S2 & S1 & S2 \\
\hline \multicolumn{14}{|c|}{ Potassium levels $\left(\mathrm{K}_{2} \mathrm{O}\right)$} \\
\hline $96 \mathrm{~kg} / \mathrm{f}$ & & 266.20 & 280.71 & 295.60 & 307.53 & 2.29 & 2.37 & 3.11 & 3.39 & 539.21 & 548.97 & 548.97 & 559.46 \\
\hline $72 \mathrm{~kg} / \mathrm{f}$ & & 225.66 & 241.62 & 257.51 & 268.44 & 2.10 & 2.18 & 2.97 & 3.15 & 510.99 & 522.68 & 519.84 & 533.16 \\
\hline $48 \mathrm{~kg} / \mathrm{f}$ & & 170.79 & 187.29 & 192.35 & 206.03 & 1.65 & 1.59 & 2.70 & 2.57 & 493.48 & 497.17 & 504.85 & 513.98 \\
\hline LSD & & 11.67 & 12.80 & 10.48 & 5.79 & 0.22 & 0.25 & 0.25 & 0.16 & 5.34 & 6.89 & 4.58 & 6.67 \\
\hline \multicolumn{14}{|c|}{ Foliar spray macro and micronutrients. } \\
\hline Contro & & 180.36 & 193.51 & 208.94 & 220.33 & 1.88 & 1.69 & 2.82 & 2.55 & 453.91 & 467.45 & 470.67 & 481.14 \\
\hline $2 \mathrm{~g} / 1$ & & 207.56 & 224.51 & 231.27 & 244.66 & 1.80 & 1.94 & 2.59 & 2.95 & 479.22 & 481.64 & 487.00 & 499.52 \\
\hline $4 \mathrm{~g} / 1$ & & 225.82 & 241.62 & 253.93 & 264.22 & 1.99 & 2.06 & 2.98 & 3.12 & 538.81 & 546.12 & 546.72 & 554.69 \\
\hline $6 \mathrm{~g} / 1$ & & 269.79 & 286.51 & 299.81 & 313.45 & 2.38 & 2.51 & 3.31 & 3.53 & 586.30 & 596.55 & 593.83 & 606.79 \\
\hline LSD & & 5.50 & 5.46 & 6.47 & 5.49 & 0.19 & 0.19 & 0.16 & 0.12 & 10.81 & 14.21 & 11.43 & 13.32 \\
\hline \multicolumn{14}{|c|}{ Interactions:K levels\& Foliar spray } \\
\hline & Control & 220.23 & 229.96 & 246.56 & 256.78 & 2.06 & 2.03 & 2.95 & 2.96 & 473.74 & 489.96 & 490.20 & 498.20 \\
\hline 96 & & 252.44 & 269.62 & 280.16 & 296.44 & 2.14 & 2.20 & 2.93 & 3.26 & 493.44 & 502.74 & 500.97 & 513.00 \\
\hline \multirow[t]{3}{*}{$\mathrm{kg} / \mathrm{fed}$} & $4 \mathrm{~g} / 1$ & 278.22 & 292.96 & 308.89 & 319.78 & 2.23 & 2.31 & 2.98 & 3.40 & 593.65 & 601.09 & 601.18 & 610.33 \\
\hline & $6 \mathrm{~g} / 1$ & 313.94 & 330.29 & 346.79 & 357.11 & 2.73 & 2.94 & 3.58 & 3.93 & 596.00 & 602.09 & 603.53 & 616.33 \\
\hline & Control & 191.34 & 207.29 & 224.27 & 234.11 & 1.88 & 1.81 & 2.85 & 2.60 & 459.00 & 473.20 & 470.85 & 486.44 \\
\hline 72 & $2 \mathrm{~g} / 1$ & 213.32 & 225.62 & 241.45 & 252.44 & 1.85 & 2.15 & 2.46 & 3.16 & 485.83 & 496.42 & 494.36 & 508.31 \\
\hline \multirow[t]{3}{*}{$\mathrm{kg} /$ fed } & $4 \mathrm{~g} / 1$ & 226.03 & 238.96 & 256.87 & 267.44 & 2.23 & 2.22 & 3.23 & 3.23 & 512.81 & 520.21 & 520.31 & 528.79 \\
\hline & $6 \mathrm{~g} / 1$ & 271.96 & 294.62 & 307.47 & 319.76 & 2.45 & 2.55 & 3.35 & 3.63 & 586.33 & 600.88 & 593.86 & 609.12 \\
\hline & Control & 129.51 & 143.29 & 156 & 170.11 & 1.72 & 1.23 & 2.68 & 2.10 & 429.00 & 439.20 & 450.96 & 458.77 \\
\hline 48 & $2 \mathrm{~g} / 1$ & 156.94 & 178.29 & 172.21 & 185.11 & 1.40 & 1.47 & 2.38 & 2.43 & 458.39 & 445.76 & 465.66 & 477.25 \\
\hline \multirow[t]{2}{*}{$\mathrm{kg} /$ fed } & $4 \mathrm{~g} / 1$ & 173.23 & 192.96 & 196.04 & 205.44 & 1.52 & 1.64 & 2.73 & 2.73 & 509.98 & 517.05 & 518.68 & 524.96 \\
\hline & $6 \mathrm{~g} / 1$ & 223.47 & 234.62 & 245.17 & 263.48 & 1.97 & 2.03 & 3.01 & 3.03 & 576.57 & 586.70 & 584.10 & 594.94 \\
\hline L.S.D. & at $5 \%$ & 9.54 & 9.46 & 11.21 & 9.51 & 0.34 & 0.32 & 0.29 & 0.21 & 18.73 & 24.62 & 19.80 & 23.07 \\
\hline
\end{tabular}


Hala A. El-Sayed et al.

2- Yield and quality parameters:

Obtained results in Tables 3 and 4 indicated that the highest values of yield parameters (total yield, grading) and quality parameters (TSS, dry matter, specific gravity and ascorbic acid) were obtained from plants fertilized by potassium level of $96 \mathrm{Kg} \mathrm{K}_{2} \mathrm{O} /$ fed followed by $72 \mathrm{Kg} /$ fed in both seasons. The positive effect of K-levels in improving total yield and its components may be imputed to the fact that K-element is one of the most important element for potato plants.

Table 3. yield and components characters of potato plants as affected by potassium levels, foliar spray with macro and micronutrients and their interactions during 2014/2015 (S1) and 2005/2006 (S2) winter seasons.

\begin{tabular}{|c|c|c|c|c|c|c|c|c|c|}
\hline \multirow{3}{*}{\multicolumn{2}{|c|}{$\begin{array}{l}\text { Characters } \\
\text { Treatments }\end{array}$}} & \multirow{2}{*}{\multicolumn{2}{|c|}{ Total yield (ton/fed) }} & \multicolumn{6}{|c|}{ Grading } \\
\hline & & & & \multicolumn{2}{|c|}{ Tuber > $60 \mathrm{~mm}(\%)$} & \multicolumn{2}{|c|}{ Tuber 30-60 mm (\%) } & \multicolumn{2}{|c|}{ Tuber $<30 \mathrm{~mm}(\%)$} \\
\hline & & S1 & S2 & S1 & S2 & S1 & S2 & S1 & S2 \\
\hline \multicolumn{10}{|c|}{ Potassium levels $\left(\mathrm{K}_{2} \mathrm{O}\right)$ : } \\
\hline \multicolumn{2}{|c|}{$96 \mathrm{~kg} / \mathrm{fed}$} & 10.46 & 11.14 & 55.12 & $56.75^{2}$ & 40.92 & 40.95 & 8.60 & 9.23 \\
\hline \multicolumn{2}{|c|}{$72 \mathrm{~kg} / \mathrm{fed}$} & 9.27 & 10.06 & 54.84 & 56.47 & 40.64 & 40.67 & 8.32 & 8.95 \\
\hline \multicolumn{2}{|c|}{$48 \mathrm{~kg} / \mathrm{fed}$} & 9.36 & 9.73 & 53.96 & 55.59 & 39.76 & 39.79 & 7.44 & 8.07 \\
\hline \multicolumn{2}{|c|}{ LSD at $5 \%$} & 0.37 & 0.23 & 0.12 & 0.065 & 0.107 & 0.18 & 0.12 & 0.09 \\
\hline \multicolumn{10}{|c|}{ Foliar spray macro and micronutrients. } \\
\hline \multicolumn{2}{|c|}{ Control } & 7.78 & 8.55 & 53.11 & 54.74 & 38.91 & 38.94 & 6.59 & 7.22 \\
\hline \multicolumn{2}{|c|}{$2 \mathrm{~g} / 1$} & 8.40 & 9.22 & 54.29 & 55.92 & 40.09 & 40.12 & 7.77 & 8.40 \\
\hline \multicolumn{2}{|l|}{$4 \mathrm{~g} / 1$} & 10.64 & 11.13 & 54.95 & 56.58 & 40.75 & 40.78 & 8.43 & 9.06 \\
\hline \multicolumn{2}{|l|}{$6 \mathrm{~g} / 1$} & 11.96 & 12.34 & 56.20 & 57.83 & 42.00 & 42.03 & 9.68 & 10.31 \\
\hline \multicolumn{2}{|c|}{ LSD at $5 \%$} & 0.12 & 0.27 & 0.18 & 0.16 & 0.13 & 0.16 & 0.074 & 0.052 \\
\hline \multicolumn{10}{|c|}{ Interactions:K levels\& Foliar spray } \\
\hline & Control & 8.99 & 9.74 & 53.93 & 55.56 & 39.73 & 39.76 & 7.41 & 8.04 \\
\hline 96 & $2 \mathrm{~g} / 1$ & 9.81 & 10.64 & 54.55 & 56.18 & 40.35 & 40.38 & 8.03 & 8.66 \\
\hline \multirow[t]{3}{*}{$\mathrm{kg} / \mathrm{fed}$} & $4 \mathrm{~g} / 1$ & 10.99 & 11.63 & 55.31 & 56.94 & 41.11 & 41.14 & 8.79 & 9.42 \\
\hline & $6 \mathrm{~g} / 1$ & 12.06 & 12.54 & 56.69 & 58.32 & 42.49 & 42.52 & 10.17 & 10.8 \\
\hline & Control & 7.23 & 8.30 & 53.63 & 55.26 & 39.43 & 39.46 & 7.11 & 7.74 \\
\hline 72 & $2 \mathrm{~g} / 1$ & 7.51 & 8.58 & 54.34 & 55.97 & 40.14 & 40.17 & 7.82 & 8.45 \\
\hline \multirow{2}{*}{$\mathrm{kg} / \mathrm{fed}$} & $4 \mathrm{~g} / 1$ & 10.41 & 10.98 & 54.96 & 56.59 & 40.76 & 40.79 & 8.44 & 9.07 \\
\hline & $6 \mathrm{~g} / 1$ & 11.91 & 12.36 & 56.42 & 58.05 & 42.22 & 42.25 & 9.90 & 10.53 \\
\hline & Control & 7.13 & 7.61 & 51.78 & 53.41 & 37.58 & 37.61 & 5.26 & 5.89 \\
\hline 48 & $2 \mathrm{~g} / 1$ & 7.87 & 8.43 & 53.98 & 55.61 & 39.78 & 39.81 & 7.46 & 8.09 \\
\hline \multirow{2}{*}{$\mathrm{kg} / \mathrm{fed}$} & $4 \mathrm{~g} / 1$ & 10.52 & 10.77 & 54.58 & 56.21 & 40.38 & 40.41 & 8.06 & 8.69 \\
\hline & $6 \mathrm{~g} / 1$ & 11.91 & 12.11 & 55.50 & 57.13 & 41.30 & 41.33 & 8.98 & 9.61 \\
\hline \multicolumn{2}{|c|}{ L.S.D. at $5 \%$} & 0.21 & 0.48 & 0.30 & 0.27 & 0.19 & 0.27 & 0.25 & 0.31 \\
\hline
\end{tabular}

Table 4. yield and component characters of potato plants as affected by potassium levels, foliar spray with macro and micronutrients and their interactions during 2014/2015 (S1) and 2005/2006 (S2) winter seasons.

\begin{tabular}{|c|c|c|c|c|c|c|c|c|c|}
\hline \multirow{2}{*}{\multicolumn{2}{|c|}{$\begin{array}{l}\text { Characters } \\
\text { Treatments } \\
\end{array}$}} & \multicolumn{2}{|c|}{ Tss(\%) } & \multicolumn{2}{|c|}{ Dry matter (\%) } & \multicolumn{2}{|c|}{ specific gravity (\%) } & \multicolumn{2}{|c|}{ Ascorbic acid (mg/g F.Wt.) } \\
\hline & & S1 & S2 & S1 & S2 & S1 & S2 & S1 & S2 \\
\hline \multicolumn{10}{|c|}{ Potassium levels $\left(\mathrm{K}_{2} \mathrm{O}\right)$ : } \\
\hline \multicolumn{2}{|c|}{$96 \mathrm{~kg} / \mathrm{fed}$} & 3.79 & 4.91 & 21.95 & 22.65 & 1.36 & 1.12 & 19.95 & 21.39 \\
\hline \multicolumn{2}{|c|}{$72 \mathrm{~kg} / \mathrm{fed}$} & 3.70 & 5.00 & 20.90 & 21.60 & 1.24 & 1.09 & 19.52 & 20.87 \\
\hline \multicolumn{2}{|c|}{$48 \mathrm{~kg} / \mathrm{fed}$} & 3.50 & 4.75 & 20.44 & 21.14 & 1.18 & 1.05 & 19.14 & 20.36 \\
\hline \multicolumn{2}{|c|}{ LSD at $5 \%$} & 0.67 & 0.64 & 0.34 & 0.29 & 0.06 & 0.03 & 0.16 & 0.11 \\
\hline \multicolumn{10}{|c|}{ Foliar spray macro and micronutrients. } \\
\hline \multicolumn{2}{|c|}{ Control } & 3.22 & 5.00 & 20.26 & 20.96 & 1.11 & 1.05 & 17.43 & 18.57 \\
\hline \multicolumn{2}{|l|}{$2 \mathrm{~g} / 1$} & 3.55 & 4.77 & 20.58 & 21.28 & 1.21 & 1.07 & 18.81 & 20.08 \\
\hline \multicolumn{2}{|l|}{$4 \mathrm{~g} / 1$} & 3.72 & 4.88 & 21.11 & 21.81 & 1.27 & 1.09 & 20.21 & 21.63 \\
\hline & 4.16 & 4.88 & 22.43 & 23.13 & 1.44 & 1.14 & 21.69 & 23.22 \\
\hline \multicolumn{2}{|c|}{$\begin{array}{l}6 \mathrm{~g} / 1 \\
\mathrm{LSD} \text { at } 5 \%\end{array}$} & 0.33 & 0.23 & 0.57 & 0.43 & 0.06 & 0.01 & 0.11 & 0.17 \\
\hline \multicolumn{10}{|c|}{ Interactions:K levels\& Foliar spray } \\
\hline \multirow{4}{*}{$\begin{array}{l}96 \\
\mathrm{~kg} / \mathrm{fed}\end{array}$} & Control & 3.33 & 5.00 & 20.97 & 21.67 & 1.16 & 1.08 & 17.86 & 19.08 \\
\hline & $2 \mathrm{~g} / 1$ & 3.66 & 4.66 & 21.53 & 22.23 & 1.28 & 1.09 & 19.24 & 20.63 \\
\hline & $4 \mathrm{~g} / 1$ & 3.83 & 5.00 & 21.94 & 22.64 & 1.32 & 1.12 & 20.63 & 22.12 \\
\hline & $6 \mathrm{~g} / 1$ & 4.33 & 5.00 & 23.35 & 24.05 & 1.67 & 1.19 & 22.06 & 23.75 \\
\hline \multirow{4}{*}{$\begin{array}{l}72 \\
\mathrm{~kg} / \mathrm{fed}\end{array}$} & Control & 3.33 & 5.00 & 20.05 & 20.75 & 1.11 & 1.05 & 17.44 & 18.57 \\
\hline & $2 \mathrm{~g} / 1$ & 3.66 & 5.00 & 20.28 & 20.98 & 1.23 & 1.06 & 18.72 & 20.07 \\
\hline & $4 \mathrm{~g} / 1$ & 3.66 & 5.00 & 20.86 & 21.56 & 1.27 & 1.10 & 20.22 & 21.66 \\
\hline & $6 \mathrm{~g} / 1$ & 4.16 & 5.00 & 22.40 & 23.10 & 1.36 & 1.16 & 21.71 & 23.20 \\
\hline \multirow{4}{*}{$\begin{array}{l}48 \\
\mathrm{~kg} / \mathrm{fed}\end{array}$} & Control & 3.00 & 5.00 & 19.76 & 20.46 & 1.06 & 1.02 & 16.99 & 18.08 \\
\hline & $2 \mathrm{~g} / 1$ & 3.33 & 4.66 & 19.93 & 20.63 & 1.13 & 1.04 & 18.48 & 19.53 \\
\hline & $4 \mathrm{~g} / 1$ & 3.66 & 4.66 & 20.53 & 21.23 & 1.23 & 1.06 & 19.77 & 21.12 \\
\hline & $6 \mathrm{~g} / 1$ & 4.00 & 4.66 & 21.55 & 22.25 & 1.30 & 1.09 & 21.31 & 22.72 \\
\hline \multicolumn{2}{|c|}{ L.S.D. at $5 \%$} & 0.58 & 0.40 & 0.99 & 0.78 & 0.11 & 0.02 & 0.20 & 0.30 \\
\hline
\end{tabular}


It is helps in producing stocky plants and healthy in appearance with thick leaves and larger tubers. In addition, it has an indispensable role in translocation of synthesized carbohydrates from plant leaves towards tubers. The obtained results are in accordance with those of Byiu and Ray (2002), Abdelgader et al. (2003) and Al-Moshileh and Errebi (2004), they noted that the total tuber yield and quality parameters were increased with increasing potassium application.

Regarding to the effect of foliar spray with macro and micronutrients on the previously mentioned characters, results in Tables 3 and 4 showed that the highest values were obtained from plants sprayed with macro and micronutrients at the rate of $6 \mathrm{~g} / \mathrm{L}$ ) in both seasons. Similar results were obtained by Gunadi (2009), Awad et al. (2010) and Ghosh et al. (2017).

Similarly, the interaction between potassium levels and foliar spray with macro and micronutrients had significant effect on yield and Its quality parameters, the best results were obtained from plants received potassium at the level of $96 \mathrm{~kg} \mathrm{~K} 2 \mathrm{O} /$ fed and sprayed with macro and micronutrientsat the rate of $6 \mathrm{~g} / \mathrm{L}$ in both seasons. Similar results were obtained by Moinuddin et al. (2005).

\section{Chemical composition:}

Results in Tables (5) showed that the highest values of $\mathrm{N} \%, \mathrm{P} \%, \mathrm{~K} \%$, Fe ppm, Zn ppm, Mn ppm and Cu ppm of potato tuber were obtained from plants fertilized with the highest level of potassium sulphate $\left(96 \mathrm{Kg} \mathrm{K}_{2} \mathrm{O} / \mathrm{fed}\right)$ followed by $72 \mathrm{Kg} \mathrm{K}_{2} \mathrm{O}$ /fed and $48 \mathrm{Kg} \mathrm{K}_{2} \mathrm{O} /$ fed in both seasons. Similar results were obtained by Abd El-Latif et al. (2011) found that $\mathrm{N}$ content in tuber has increased significantly by adding $96 \mathrm{k}_{2} \mathrm{O} \mathrm{kg} / \mathrm{fed}$., while, $\mathrm{P}$ content has increased when $72 \mathrm{~K}_{2} \mathrm{O} \mathrm{kg} / \mathrm{fed}$. was applied, at the same time as $\mathrm{K}$ content in tuber has increased by adding the high level of $\mathrm{K}$ fertilizers $\left(120 \mathrm{~K}_{2} \mathrm{O} \mathrm{kg} / \mathrm{fed}\right.$.). On the other hand, the lowest $\mathrm{N}, \mathrm{P}$ and $\mathrm{K}$ contents were recorded when control (without addition of $\mathrm{k}$ fertilizers) in both seasons.

Table 5. Chemical characters of potato plants as affected by potassium levels, foliar spray with macro and micronutrients and their interactions during 2014/2015 (S1) and 2005/2006 (S2) winter seasons.

\begin{tabular}{|c|c|c|c|c|c|c|c|c|c|c|c|c|c|c|c|}
\hline \multirow{2}{*}{\multicolumn{2}{|c|}{$\begin{array}{l}\text { Characters } \\
\text { Treatments }\end{array}$}} & \multicolumn{2}{|c|}{ N\% } & \multicolumn{2}{|c|}{$\mathbf{P \%}$} & \multicolumn{2}{|c|}{$\mathbf{K} \%$} & \multicolumn{2}{|c|}{ Fe ppm } & \multicolumn{2}{|c|}{ Zn ppm } & \multicolumn{2}{|c|}{ Mn ppm } & \multicolumn{2}{|c|}{ Cu ppm } \\
\hline & & S1 & S2 & S1 & S2 & S1 & S2 & S1 & S2 & S1 & S2 & S1 & S2 & S1 & S2 \\
\hline \multicolumn{16}{|c|}{ Potassium levels $\left(\mathrm{K}_{2} \mathrm{O}\right)$ : } \\
\hline \multicolumn{2}{|c|}{$96 \mathrm{~kg} / \mathrm{fed}$} & 1.66 & 1.81 & 0.232 & 0.253 & 3.19 & 3.16 & 11.14 & 12.33 & 4.90 & 5.97 & 3.75 & 4.18 & 1.37 & 1.46 \\
\hline \multicolumn{2}{|c|}{$72 \mathrm{~kg} / \mathrm{fed}$} & 1.55 & 1.68 & 0.217 & 0.243 & 2.99 & 3.05 & 10.85 & 11.95 & 4.96 & 5.80 & 3.54 & 3.95 & 1.27 & 1.35 \\
\hline \multicolumn{2}{|c|}{$48 \mathrm{~kg} / \mathrm{fed}$} & 1.46 & 1.58 & 0.214 & 0.235 & 2.62 & 2.96 & 10.48 & 11.66 & 4.77 & 5.57 & 3.31 & 3.75 & 1.18 & 1.23 \\
\hline \multicolumn{2}{|c|}{ LSD at $5 \%$} & 0.04 & 0.05 & 0.016 & 0.006 & 0.02 & 0.02 & 0.07 & 0.08 & 0.29 & 0.13 & 0.03 & 0.02 & 0.04 & 0.02 \\
\hline \multicolumn{16}{|c|}{ Foliar spray macro and micronutrients. } \\
\hline & 1.13 & 1.23 & 0.173 & 0.201 & 2.40 & 2.58 & 9.37 & 10.55 & 3.78 & 5.02 & 2.57 & 3.05 & 0.88 & 0.79 \\
\hline \multicolumn{2}{|l|}{$2 \mathrm{~g} / 1$} & 1.40 & 1.52 & 0.208 & 0.228 & 2.88 & 2.91 & 10.37 & 11.52 & 4.66 & 5.51 & 3.19 & 3.63 & 1.12 & 1.16 \\
\hline \multicolumn{2}{|l|}{$4 \mathrm{~g} / 1$} & 1.70 & 1.88 & 0.236 & 0.256 & 3.16 & 3.22 & 11.28 & 12.53 & 5.22 & 6.04 & 3.86 & 4.24 & 1.40 & 1.55 \\
\hline \multicolumn{2}{|l|}{$6 \mathrm{~g} / 1$} & 2.00 & 2.13 & 0.267 & 0.289 & 3.29 & 3.51 & 12.29 & 13.32 & 5.85 & 6.53 & 4.51 & 4.92 & 1.70 & 1.88 \\
\hline \multicolumn{2}{|c|}{ LSD at $5 \%$} & 0.04 & 0.04 & 0.013 & 0.002 & 0.03 & 0.03 & 0.05 & 0.13 & 0.27 & 0.14 & 0.03 & 0.05 & 0.02 & 0.03 \\
\hline \multicolumn{16}{|c|}{ Interactions: K levels\& Foliar spray } \\
\hline & Control & 1.22 & 1.32 & 0.190 & 0.211 & 2.50 & 2.69 & 9.69 & 10.86 & 3.25 & 5.26 & 2.78 & 3.25 & 0.97 & 0.90 \\
\hline 96 & $2 \mathrm{~g} / 1$ & 1.49 & 1.68 & 0.219 & 0.236 & 3.08 & 3.03 & 10.69 & 11.97 & 4.87 & 5.67 & 3.42 & 3.83 & 1.22 & 1.28 \\
\hline \multirow[t]{2}{*}{$\mathrm{kg} / \mathrm{fed}$} & $4 \mathrm{~g} / 1$ & 1.81 & 2.00 & 0.244 & 0.265 & 3.53 & 3.32 & 11.41 & 12.84 & 5.42 & 6.22 & 4.08 & 4.46 & 1.50 & 1.68 \\
\hline & $6 \mathrm{~g} / 1$ & 2.11 & 2.24 & 0.278 & 0.301 & 3.64 & 3.58 & 12.79 & 13.64 & 6.08 & 6.71 & 4.73 & 5.16 & 1.79 & 1.98 \\
\hline \multirow{4}{*}{$\begin{array}{l}72 \\
\mathrm{~kg} / \mathrm{fed}\end{array}$} & Control & 1.10 & 1.23 & 0.155 & 0.200 & 2.38 & 2.58 & 9.39 & 10.53 & 4.13 & 5.09 & 2.58 & 3.04 & 0.88 & 0.80 \\
\hline & $2 \mathrm{~g} / 1$ & 1.41 & 1.50 & 0.209 & 0.230 & 2.96 & 2.89 & 10.39 & 11.46 & 4.63 & 5.54 & 3.16 & 3.62 & 1.12 & 1.17 \\
\hline & $4 \mathrm{~g} / 1$ & 1.70 & 1.88 & 0.238 & 0.254 & 3.24 & 3.23 & 11.37 & 12.51 & 5.23 & 6.04 & 3.88 & 4.24 & 1.40 & 1.55 \\
\hline & $6 \mathrm{~g} / 1$ & 2.00 & 2.11 & 0.268 & 0.289 & 3.40 & 3.50 & 12.27 & 13.30 & 5.85 & 6.52 & 4.53 & 4.91 & 1.70 & 1.88 \\
\hline \multirow{4}{*}{$\begin{array}{l}48 \\
\mathrm{~kg} / \mathrm{fed}\end{array}$} & Control & 1.06 & 1.14 & 0.175 & 0.193 & 2.31 & 2.46 & 9.02 & 10.26 & 3.97 & 4.72 & 2.35 & 2.88 & 0.79 & 0.66 \\
\hline & $2 \mathrm{~g} / 1$ & 1.29 & 1.39 & 0.198 & 0.219 & 2.61 & 2.81 & 10.05 & 11.13 & 4.49 & 5.33 & 3.00 & 3.44 & 1.04 & 1.05 \\
\hline & $4 \mathrm{~g} / 1$ & 1.60 & 1.77 & 0.228 & 0.249 & 2.73 & 3.10 & 11.06 & 12.23 & 5.03 & 5.86 & 3.63 & 4.02 & 1.30 & 1.43 \\
\hline & $6 \mathrm{~g} / 1$ & 1.90 & 2.04 & 0.256 & 0.278 & 2.82 & 3.45 & 11.81 & 13.01 & 5.61 & 6.37 & 4.26 & 4.68 & 1.59 & 1.79 \\
\hline \multicolumn{2}{|l|}{ L.S.D. } & 0.07 & 0.07 & 0.023 & 0.004 & 0.06 & 0.05 & 0.10 & 0.23 & 0.47 & 0.24 & 0.06 & 0.07 & 0.04 & 0.05 \\
\hline
\end{tabular}

Also, the results in Table (5) indicate that the highest values of $\mathrm{N} \%, \mathrm{P} \%, \mathrm{~K} \%$, Fe ppm, Zn ppm, Mn ppm and $\mathrm{Cu}$ ppm of potato tuber using foliar spray with macro and micronutrients at the highest level $(6 \mathrm{~g} / \mathrm{l})$. Similar results were obtained by Horvat et al. (2014) reported that the concentration of nitrogen, phosphorus, potassium and calcium in potato tuber significantly influenced by foliar fertilizers. Moreover, Ghosh et al. (2017) showed that foliar application with potassium significantly improved quality parameters (ascorbic acid, specific gravity and reducing sugar).

In the same tables, the interaction results indicated that the Chemical character's constituents $(\mathrm{N}, \mathrm{P}, \mathrm{K}, \mathrm{Fe}, \mathrm{Zn}$, $\mathrm{Mn}, \mathrm{Cu}$ ) of potato tuber were significantly increased by using potassium at the level of $96 \mathrm{~kg} \mathrm{~K}_{2} \mathrm{O} /$ fed and spray with macro and micronutrients at the rate of $6 \mathrm{~g} / \mathrm{L}$ compared with the other interactions. Similar results were obtained by Salim et al. (2014) found that foliar spraying with potassium silicate or potassium nitrate had strong stimulating effect on mineral nutrients $(\mathrm{N}, \mathrm{P}, \mathrm{K}, \mathrm{Zn}, \mathrm{Mn}$ and $\mathrm{Fe}$ ) and protein concentration of potato leaves in both seasons.

4. storability:

Results in Table 6 indicated that the highest values of weight loss $\%$ of potato tuber were obtained from plants fertilized with potassium sulphate at $48 \mathrm{Kg} \mathrm{K} 2 \mathrm{O} / \mathrm{fed}$, while the highest values of ascorbic acid (mg/g FW) were obtained with potassium fertilization at $96 \mathrm{~kg} \mathrm{~K} 2 \mathrm{O} / \mathrm{fed}$. Similar results were obtained by Etman et al. (2002) reveal that application of $\mathrm{K}$ at two levels of 75 and $100 \mathrm{~kg}$ 
$\mathrm{K}_{2} \mathrm{O} /$ fed caused a significant decreased in percentage of total weight loss of sweet potato tuber roots compared with the other levels $\left(25-50 \mathrm{~kg} \mathrm{~K}_{2} \mathrm{O} / \mathrm{fed}\right)$. In addition, Imas and Bansal (1999) illustrated that applying $\mathrm{K}$ to potato significantly decreased weight losses from the tubers after harvest.

Regarding to the effect of foliar spray with macro and micro nutrients, data in Table 6 showed that the highest values of weight loss $\%$ were obtained when potato plants sprayed with the lowest level of foliar application while the highest values ascorbic acid of potato tubers were obtained from plants sprayed with macro and micronutrients at the rate of $6 \mathrm{~g} / \mathrm{L}$ in both seasons. Similar results were obtained by El-Sawy et al. (2000), El-Sayed et al. (2007) and El-Sawy (2011).

On the other hand, the interaction between potassium levels and foliar spray with macro and micro nutrients had the significant effect on weight loss \% and ascorbic acid (mg/g FW), the best results were obtained from plants received potassium at the level of $96 \mathrm{~kg}$ $\mathrm{K}_{2} \mathrm{O}$ /fed and sprayed with macro and micronutrients at the rate of $6 \mathrm{~g} / \mathrm{L}$ in both seasons. Similar results were obtained by El-Sawy (2011) found that application of potassium at $100 \mathrm{~kg} \mathrm{~K}_{2} \mathrm{O} / \mathrm{fed}$ and foliar of $\mathrm{K}$ at $0.5 \%$ decreased weight loss $(\%)$ decay percentage and sprouting in both seasons.

Table 6. Storage characters of potato plants as affected by potassium levels, foliar spray with macro and micronutrients and their interactions during 2014/2015 (S1) and 2005/2006 (S2) winter seasons.

\begin{tabular}{|c|c|c|c|c|c|c|c|c|c|}
\hline \multirow{3}{*}{\multicolumn{2}{|c|}{$\begin{array}{l}\text { Characters } \\
\text { Treatments }\end{array}$}} & \multirow{2}{*}{\multicolumn{2}{|c|}{$\begin{array}{c}\text { Ascorbic acid } \\
\text { mg/g FW }\end{array}$}} & \multicolumn{6}{|c|}{ Weight loss \% } \\
\hline & & & & \multicolumn{2}{|c|}{60 days } & \multicolumn{2}{|c|}{120 days } & \multicolumn{2}{|c|}{180 days } \\
\hline & & S1 & S2 & S1 & S2 & S1 & S2 & S1 & S2 \\
\hline \multicolumn{10}{|c|}{ Potassium levels $\left(\mathrm{K}_{2} \mathrm{O}\right)$ : } \\
\hline \multicolumn{2}{|c|}{$96 \mathrm{~kg} / \mathrm{fed}$} & 8.18 & 8.82 & 1.08 & 1.28 & 7.89 & 8.08 & 22.91 & 23.10 \\
\hline \multicolumn{2}{|c|}{$72 \mathrm{~kg} / \mathrm{fed}$} & 7.99 & 8.61 & 1.18 & 1.38 & 7.98 & 8.18 & 23.00 & 23.20 \\
\hline \multicolumn{2}{|c|}{$48 \mathrm{~kg} / \mathrm{fed}$} & 7.80 & 8.44 & 1.27 & 1.46 & 8.07 & 8.27 & 23.09 & 23.29 \\
\hline \multicolumn{2}{|c|}{ LSD at $5 \%$} & 0.07 & 0.11 & 0.07 & 0.02 & 0.07 & 0.03 & 0.039 & 0.054 \\
\hline \multicolumn{10}{|c|}{ Foliar spray macro and micronutrients. } \\
\hline \multicolumn{2}{|c|}{ Control } & 7.14 & 7.74 & 1.45 & 1.66 & 9.64 & 9.85 & 24.66 & 24.87 \\
\hline \multicolumn{2}{|l|}{$2 \mathrm{~g} / 1$} & 7.68 & 8.31 & 1.20 & 1.38 & 8.50 & 8.68 & 23.52 & 23.70 \\
\hline \multicolumn{2}{|l|}{$4 \mathrm{~g} / 1$} & 8.29 & 8.91 & 1.09 & 1.28 & 7.43 & 7.62 & 22.45 & 22.64 \\
\hline & 8.85 & 9.54 & 0.97 & 1.17 & 6.35 & 6.55 & 21.37 & 21.57 \\
\hline \multicolumn{2}{|c|}{$\begin{array}{l}6 \mathrm{~g} / 1 \\
\mathrm{LSD} \text { at } 5 \%\end{array}$} & 0.09 & 0.10 & 0.017 & 0.09 & 0.03 & 0.08 & 0.04 & 0.02 \\
\hline \multicolumn{10}{|c|}{ Interactions:K levels\& Foliar spray } \\
\hline & Control & 7.33 & 7.95 & 1.27 & 1.46 & 9.46 & 9.65 & 24.48 & 24.67 \\
\hline 96 & $2 \mathrm{~g} / \mathrm{l}$ & 7.85 & 8.51 & 1.13 & 1.33 & 8.43 & 8.63 & 23.45 & 23.65 \\
\hline \multirow[t]{3}{*}{$\mathrm{kg} / \mathrm{fed}$} & $4 \mathrm{~g} / 1$ & 8.50 & 9.09 & 1.04 & 1.23 & 7.38 & 7.57 & 22.4 & 22.59 \\
\hline & $6 \mathrm{~g} / 1$ & 9.04 & 9.72 & 0.91 & 1.11 & 6.29 & 6.49 & 21.31 & 21.51 \\
\hline & Control & 7.14 & 7.72 & 1.50 & 1.71 & 9.69 & 9.90 & 24.71 & 24.92 \\
\hline 72 & $2 \mathrm{~g} / 1$ & 7.70 & 8.31 & 1.2 & 1.37 & 8.5 & 8.67 & 23.52 & 23.69 \\
\hline \multirow[t]{2}{*}{$\mathrm{kg} / \mathrm{fed}$} & $4 \mathrm{~g} / 1$ & 8.28 & 8.92 & 1.08 & 1.28 & 7.42 & 7.62 & 22.44 & 22.64 \\
\hline & $6 \mathrm{~g} / 1$ & 8.86 & 9.52 & 0.94 & 1.15 & 6.32 & 6.53 & 21.34 & 21.55 \\
\hline \multirow{4}{*}{$\begin{array}{l}48 \\
\mathrm{~kg} / \text { fed }\end{array}$} & Control & 6.95 & 7.55 & 1.6 & 1.81 & 9.79 & 10 & 24.81 & 25.02 \\
\hline & $2 \mathrm{~g} / 1$ & 7.50 & 8.10 & 1.27 & 1.45 & 8.57 & 8.75 & 23.59 & 23.77 \\
\hline & $4 \mathrm{~g} / 1$ & 8.10 & 8.72 & 1.15 & 1.34 & 7.49 & 7.68 & 22.51 & 22.70 \\
\hline & $6 \mathrm{~g} / 1$ & 8.65 & 9.37 & 1.06 & 1.27 & 6.44 & 6.65 & 21.46 & 21.67 \\
\hline \multicolumn{2}{|c|}{ L.S.D. at $5 \%$} & 0.16 & 0.17 & 0.06 & 0.04 & 0.02 & 0.06 & 0.018 & 0.04 \\
\hline
\end{tabular}

\section{CONCLUSION}

In view of the obtained and discussed results, it was found that fertilization with potassium at $96 \mathrm{~kg} \mathrm{~K}_{2} \mathrm{O}$ and foliar application of macro and micronutrients at $6 \mathrm{~g} / \mathrm{l}$ gave high vegetative growth parameters and produced higher yield with high quality parameters under the conditions of this study.

\section{REFERENCES}

A.O.A.C. (1975). Official Method of Analysis of the Association Official Analytical Chemists. 12th Ed. Published by the association of official analytical chemists.

Abd El-Latif, K.M.; E.A.M. Osman; R. Abdullah and N. Abd El Kader (2011). Response of potato plants to potassium fertilizer rates and soil moisture deficit. Advances in Applied Science Research, 2: 388397.
Abdelgader, A. H.; M. A. Errebhi; H. M. Al Sarhan and M. Ibrahim (2003). The effect of different levels of additional potassium on yield and industrial qualities of potato (Solanum tuberosum L.) in an irrigated arid region. Amer. J. of Potato Res., 80: 219- 222.

Abdel-Hadi, A. H.; K. G.; H. W. Doeriny; M. S. Kadr; Y. H. Mohamed; A. A. Moustafa; M. E. Taha and A. Alexander (1986). Effect of foliar fertilization on crops under Egyptian conditions. Foliar fertilization developments in plant and soil sciences. Dordrecht, Netherlands, Marttinus Nijhoff Publishers, PP. 126141.

Allison, M. F. J. H. Foeler and E. J. Allen (2001). Responses of potato (Solanum tuberosum L.) to potassium fertilizers. J. of Agric. Sci. Cambridge., 136: 407- 426. 
Al-Moshileh, A. M. and M. A. Errebi (2004). Effect of various potassium sulfate rates on growth, yield and quality of potato grown under sandy soil and arid condition. IPI regional workshop on potassium and fertigation development in West Asia and North Africa, Rabat, Morocco 24- 28 November. (C. F. computer search).

Awad, El. M. M.; R. A. Mohamed and H. Asfoure (2010). Effect of compost, foliar spraying with potassium and boron on growth, yield and fruit quality of strawberry. J. plant production, Mansoura. Univ. 1(8): 1101- 1112.

Byiu, G. and R. C. Ray (2002). Effect of graded levels of potassium on yield and yield components of Sweet potato. Orissa J. Hort., 30 (2): 91- 93.

Dkhil, B.B.; M. Denden and S. Aboud (2011). Foliar potassium fertilization and its effect on growth, yield and quality of potato grown under loam sandy soil and semi-arid conditions. International Journal of Agricultural Research, 6(7): 593-600.

Doeing, H.W. (1986). Foliar fertilization. An effected method for controlling micronutrients deficiencies on calcareous and saline soil in arid lands. In: Proc. Symp. Application of special fertilizers. Alex. 2123 Feb., Egypt.

El- Hadidi, E. M. and M. M. Mansour (2008). Effect of potassium and zinc fertilization on growth, nutrients contents, yield and quality sweet potato plant growth on clay soil. J. Agric. Sci. Mansoura Univ., 33(6): 4589- 4608.

El- Sawy, B. I.; E. A. Radwan and N. A. Hassan (2000). Growth and yield of potato as affected by soil and foliar potassium application. J. Agric. Sci. Mansoura Univ., 25(9): 5843- 5850.

El- Sawy, M. B. I. (2011). Effect of soil fertilization and foliar spray of potassium on quality and storage ability of sweet potato. J. Agric. Sci. Mansoura. Univ., 2(3): 453- 466.

El- Sayed, H. A.; A. H. A. El-Morsy and H. M. B. ElMetwally (2007). Effect of some organic fertilization sources and micronutrients application methods on yield and quality of potato (Solanum tuberosum L.). J. Agric. Sci. Mansoura. Univ., 31(9) 7533- 7546.

El- Sirafy Z. M.; A. Khadra-Abbady; A. M. El-Ghamry and R. A. El-Dissoky (2008). Potato yield quality, quantity and profitability as affected by soil and foliar potassium application. Res. J. Agric. And Biological Sci., 4(6): 912- 922.

Eleiwa M.E.; S.A. Ibrahim and M.F. Mohamed (2012). Combined effect of NPK levels and foliar nutritional compounds on growth and yield parameters of potato plants (Solanum tuberosum L.). African Journal of Microbiology Research, 6 (24) 5100-5109.

Etman, A. A.; N. A Hassan; M. M. Saffan and M. A. Sharaf El-Din (2002). Studies on the influence of fertilization and cutting planting methods on chemical constituents and storage ability of sweet potato. 2nd inter. Conf. Hort. Sci. 10- 12. Sept. 2002. J. Agric. Res., Fac., Agric Kafrelshsikh, Tanta Univ., Egypt, 28(3/1): 131- 141.
Ewais, Magda A.; D. A. Sayed and A. A. Khalil (2010). Effect of application methods of potassium and some micronutrients on yield and quality of potato. J. of soil Sci. and Agric. Eng., 1(3): 211- 223.

Ghosh, D.; S. Sakar; K. Brahmachari; S. Garai; M. Pal and A. Sharma (2017). Potassium schoenite: an emerging source of potassium for improving growth, yield and quality of potato. J. of Exp. Bio. and Agric. Sci., 5(2): 173- 182.

Gomez, K. A. and A. A. Gomez (1984). Statistical procedures for agriculture research (2nd ed). An International Rice Research Book. Wiley Interscience Publication, John Wiley and Sons, New York. 680 pp.

Gunadi, N. (2009). Response of potato to potassium fertilizer sources and application methods in andisols of west java. Indonesian J. Agric. Sci., 10(2): 65- 72 .

Habib, H. A. M.; M. R. Shafeek; M. F. Zaki and Z. S. ElShal (2011). Response of potato plants (Solanum tuberosum L.) to foliar application with different sources of potassium. Inter. J. Academic Res., 3(3): 129- 132.

Horvat, T.; M. Poljak; B. Lazarevic; Z. Svecnjak and K. Hanacek (2014). Effect of foliar fertilizers on physiological characteristics of potato. Romanian Agric. Res., 31: 159- 165.

Imas P. and S. Bansal (1999). Potassium and Integrated Nutrient Management in Potato. Presented at the Global Conference on Potato. New Delhi, INDIA., 6-11 December (C.F. computer search)

Jackson, M. L. (1967). Soil Chemical Analysis. PrenticeHall of India, Private Limited, New Delhi, pp. 111204.

Kumar, S.P.; C.V. Kumar and B. Bandana (2010). Effects of salicylic acid on seedling growth and nitrogen metabolism in cucumber (Cucumis sativus L) Journal of Stress Physiology \& Biochemistry, 6(3): 102- 113.

Mahmoud, A. R. and M. M. Hafez (2010). Increasing productivity of potato plants (Solanum tuberosum L.) By using potassium fertilizer and humic acid application, Inter. J. Academic Res., 2: 83- 88.

Marchand, M.; B. Bourrie; D. Anac and P. Martin (1999), Use of potash fertilizers through different application methods for high yield and quality of crops. Imp. Crop Qual. Nutri. Mang., 5: 13-17.

Marschner, H., (1995). Mineral Nutrition of Higher Plants. (Academic Press Ltd.: London). Marschner, H., 2002. Mineral nutrition of higher plants. 2nd ed. Academic Press, Londres. Marschner, P., 2012. Mineral Nutrition of Higher Plants, 3rd ed.; Academic Press: London, UK; pp. 178-189.

Medvedev, A. G.; N. K. Chertko; G. A. Lipskaya; N. P. Lvanov; A. A. Bubnov; L. Y. Suirnovskii and L. E. Skyrko (1981). Effect of trace elements on yield of potatoes grown on reclaimed dernopodzolic grey soils. Agrokhimiya, 3:109- 114. (C. F. Potato Abst., 8:9). 
Moinuddin, S. U.; SINGH and S. K. BANSAL (2005). Growth, yield, and economics of potato in relation to progressive application of potassium fertilizer. Journal of Plant Nutrition, 28(1): 182-200.

Prummel, G. (1978). Effect of phosphate and potassium fertilizing on the grading of potatoes. Repport institute voor Bodemuruchtbaarheid, 16-78, $11 \mathrm{pp}$. (C.F. Potato Abst., 7:6).

Rashid, A.; H. Abdul and Nawabali. (1989). Effect of copper and zinc alone or in combination, on the growth and yield of potato. Sarhad J. Agric., 5(5): 521- 525.

Regmi, A. P.; Ladha, J. K.; E. Pasuquin; H. Pathak; P. R. Hobbs; L. L. Shrestha; D. B. Gharti; and E. Duveiller (2002). The role of Potassium in sustaining yields in a long-term rice-wheat experiment in the Indo-Gangetic plains of Nepal. Biol. Fert. Soils 36:240-247.

Roemheld, V. and M. M. El-Fouly (1999). Foliar nutrient application challenge and limits in crop production. Proceedings of the 2nd International Workshop on Foliar Fertilization, Bangkok, Thailand, 4-10.
Salim, B. B. M.; H. G. Abd El-Gawad and A. Abou ElYazied (2014). Effect of foliar spray of different potassium sources on growth yield and mineral composition of potato (Solanum tuberosum L.) Middle East J. of Applied Sci., 4(4): 1197- 1204.

Singh, J. and S.K. Bansal (2000). Relative effect of two sources of potassium on yield and economics of potato production in an Inceptisol of western UP. Journal of Potassium Research, 16(1/4): 52-54.

Stashauskaite, S.; U. Vonsavichene; G. Navaitene and E. Shltenene (1979). Dependence of plant growth on conditions and accumulation of mineral elements. Liet TSR Aukstuiu Mokuklu Mokslo Darbai Biologiya. 17:49- 59.

Taya, J. S.; Malik, Y. S.; M. L. Pandita and S. C. Khurana (1994). Fertilizer management in potato based cropping system. Growth and yield of potato. J. Indian potato Assoc., 21(3/4): 184- 188.

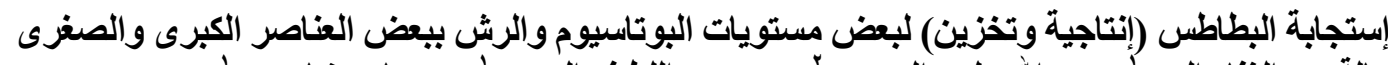

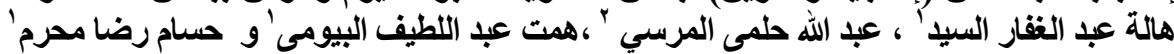

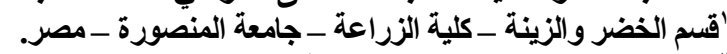

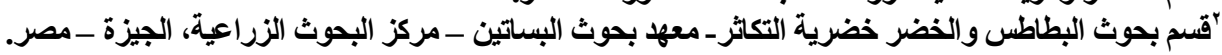

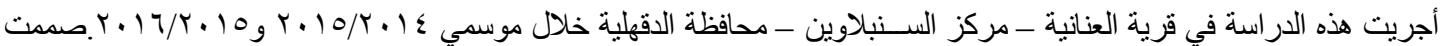

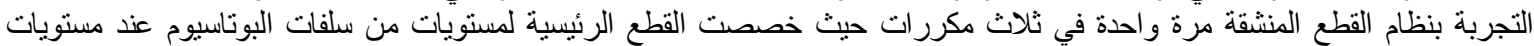

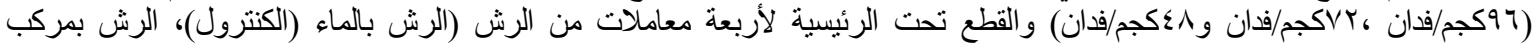

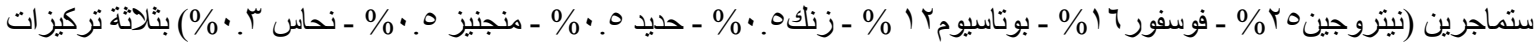

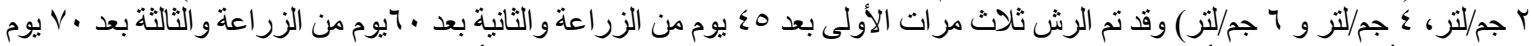

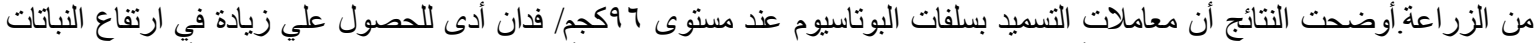

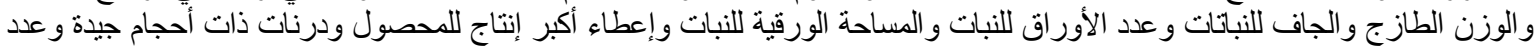

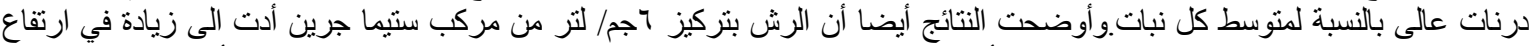

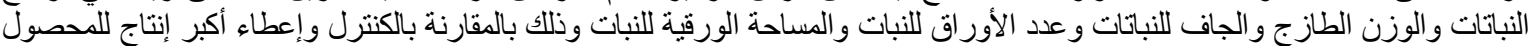

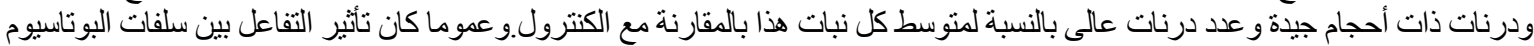

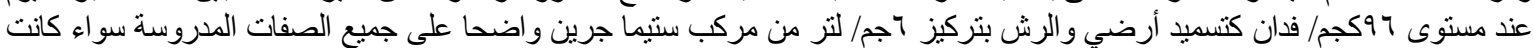
خضرية أو ثمرية أو فسيولوجية لونية
} 